\title{
ESTUDO DA UTILIZAÇÃO NO PRÉ-OPERATÓRIO DE MEDICAMENTOS OU DROGAS FITOTERÁPICAS QUE ALTERAM A COAGULAÇÃO SANGÜÍNEA
}

\author{
STUDY OF THE USE OF MEDICAMENTS OR HERBAL MEDICINES IN THE \\ PREOPERATIVE THAT ALTER THE BLOOD COAGULATION
}

\author{
Marco Willians Baena Destro ${ }^{1}$; Manlio Basílio Speranzini, TCBC-SP²; Cristina Destro ${ }^{3}$; \\ Camila Guerra $^{4}$; Guêiguila Cristina Recco ${ }^{5}$; Luís Gustavo Capochin Romagnolo ${ }^{6}$
}

\begin{abstract}
RESUMO: Objetivo: Verificar com que freqüência medicamentos elou drogas fitoterápicas que interferem na coagulação sangüínea são utilizados por pacientes que serão submetidos à intervenção cirúrgica e cujo uso não é relatado ao cirurgião; ou quando este é informado não se recomenda a sua interrupção antes da operação. Método: Estudo quantitativo com variáveis qualitativas, transversal, sobre o uso de medicamentos (AAS e Vitamina E) e fitoterápicos (Ginkgo biloba, Alho, Ginseng e Gengibre) por parte de 416 pacientes em programação pré-operatória. Desenvolvido no Hospital Universitário de Taubaté (HUT) no Hospital Regional de Taubaté (HRT) e em um Consultório Particular de Cirurgia Plástica. Estes dados foram obtidos por meio de questionário com perguntas relacionadas ao uso ou não das drogas referidas no último ano e nos 10 dias que antecediam a operação, se o cirurgião foi informado do fato e se houve recomendação médica para a sua suspensão. Resultados: do total pesquisado $58,89 \%$ não fizeram uso de alguma das drogas em questão, $39 \%$ as usaram no último ano e 13,83\% usaram nos últimos 10 dias. O AAS foi o medicamento mais utilizado e dentre os fitoterápicos, o Ginkgo biloba. Do total pesquisado 73,69\% dos pacientes não informaram ao cirurgião o uso destas drogas. Conclusões: Na população estudada é elevada a porcentagem do uso de drogas que interferem na coagulação sanguínea, sendo as mais utilizadas o AAS, a vitamina E e o Ginkgo biloba. A maioria dos pacientes pesquisados não informou ao cirurgião a sua utilização. Deve-se enfatizar a necessidade de pesquisar o uso dessas drogas no pré-operatório, com o objetivo de reduzir possíveis complicações hemorrágicas per e pós-operatórias, pelas suas complicações médicas e eventuais implicações legais (Rev. Col. Bras. Cir. 2006; 33(2): 107-111).
\end{abstract}

Descritores: Fitoterapia; Plantas medicinais; Coagulação sangüínea; Inibidores da agregação de plaquetas; Ginkgo biloba.

\section{INTRODUÇÃO}

Fitoterápicos são definidos como produtos derivados de plantas usados com propósitos medicinais e para promover a saúde ${ }^{1}$. São tidos como naturais, mas não necessariamente inócuos².

O consumo de fitoterápicos ocorre há 60.000 anos $^{3}$ e uma pesquisa recente demonstrou que cerca de $37 \%$ da população adulta dos Estados Unidos da América estão utilizando estes produtos ${ }^{4}$. Nesse país são considerados "suplementos dietéticos", enquanto que no Brasil são tidos como medicamentos pela Portaria n²2/1967 da Agência Nacional de Vigilância Sanitária e pela Resolução-RDC nº17/ $2000^{5}$.

Dentre os fitoterápicos que alteram a coagulação sanguínea, foram considerados neste estudo ginkgo biloba, alho em comprimido, ginseng e gengibre. Também foi investigado o uso dos seguintes medicamentos: vitamina E e ácido acetilsalisílico (AAS) por interferirem na coagulação sanguínea, serem de fácil acesso e de uso comum pela população em geral.

O extrato de Ginkgo biloba é utilizado na prática clínica em diversas doenças como distúrbios de memória, demência e síndrome de Alzheimer ${ }^{6,7}$, glaucoma ${ }^{8}$, distúrbios cardiovasculares, isquemia cerebral, para aumentar a viabilidade de retalhos cutâneos, para aumento da atividade e libido sexual, em doenças psiquiátricas e na depressão ${ }^{9,10}$. Seu efeito na coagulação sanguínea ocorre por inibição da agregação plaquetária induzida pela adenosina difosfato (ADP), antagonismo do efeito do fator de agregação plaquetária (FAP) e por inibição da agregação dos eritrócitos ${ }^{11}$

$\mathrm{O}$ alho tem sido reconhecido como agente benéfico em várias doenças. ${ }^{12}$, mas o seu efeito antiplaquetário aumenta o risco de sangramento pela presença do di e trisulfeto de dialila e metilalila, que inibem a tromboxano-sintetase, enzima de grande importância na formação do tromboxano $\mathrm{A}_{2}{ }^{13}$.

1. Professor Titular de Cirurgia Plástica da Universidade de Taubaté; Mestre e Doutor em Cirurgia Plástica pela Universidade Federal de Minas Gerais; Especialista em Cirurgia Plástica pela Sociedade Brasileira de Cirurgia Plástica.

2. Professor Livre-Docente do Departamento de Cirurgia da Universidade de São Paulo; Professor Titular do Departamento de Cirurgia da Universidade do ABC - São Paulo; Professor Titular do Departamento de Cirurgia da Universidade de Taubaté.

3. Médica residente(R2) de Cirurgia Geral do Hospital Regional de Taubaté.

4. Médica pela Universidade de Taubaté.

5. Médica residente de Ginecologia e Obstetrícia do Hospital Escola da Universidade de Taubaté

6. Médico pela Universidade de Taubaté.

Recebido em 03/11/2005

Aceito para publicação em 29/12/2005

Conflito de interesses: nenhum

Fonte de financiamento: nenhuma

Trabalho realizado no Hospital Escola da Universidade de Taubaté, Hospital Regional de Taubaté e Clínica Particular de Cirurgia Plástica. 
O ginseng é um fitoterápico nativo da Ásia que possui inúmeras ações terapêuticas, tais como estimulante, tônico e diurético ${ }^{14}$, aumento da vitalidade e hipoglicemiante ${ }^{15}$. O Ginseng possui como substância ativa o "ginsenosídio" ${ }^{16}$, que além dos efeitos citados acima, atua inibindo a agregação plaquetária, prolonga o tempo de coagulação da trombina e ativação parcial da tromboplastina, aumentando assim o risco de sangramento per e pós-operatório ${ }^{17}$.

O gengibre tem sido amplamente utilizado na Índia e na China como produto medicinal há pelo menos 2500 anos $^{18,19}$. Estudos "in vitro" sugerem que os efeitos antiinflamatórios do gengibre estão relacionados com a inibição do metabolismo do ácido aracdônico ${ }^{20,21}$, com diminuição da agregação plaquetária e redução da produção de tromboxano ${ }^{22}$.

A vitamina E é o nome coletivo de um grupo de tocoferóis e tocotrienóis ${ }^{23}$. Funciona prevenindo a lipoperoxidação reduzindo assim a incidência de doenças cardiovasculares $^{24}$. Em relação à coagulação sanguínea, a mesma atua diminuindo a adesividade das plaquetas ao colágeno. Salienta-se que os efeitos da vitamina $\mathrm{E}$ persistem por duas a seis semanas após a interrupção do suplemento, possivelmente por causa do tocotrienol armazenado no tecido adiposo ${ }^{25}$.

O ácido acetilsalisílico (AAS) é o fármaco de eleição para o alívio de processos dolorosos, inflamatórios agudos e crônicos e como antipirético ${ }^{26}$. Também é utilizado na prevenção, primária e secundária, de doenças cardiovasculares como: angina pectoris, infarto do miocárdio, trombose venosa e embolia pulmonar ${ }^{27}$. Interfere na coagulação sanguínea bloqueando, de forma irreversível, a cicloxigenase que existe na plaqueta em quantidade limitada por não haver produção protéica neste fragmento celular ${ }^{26}$. Assim, a ação de inibição plaquetária do AAS dura por toda a vida da plaqueta, 7 a 10 dias, potencializando o risco de sangramento neste período.

O objetivo do estudo é o de analisar, em pacientes passíveis de serem submetidos à intervenção operatória:

1. A freqüência do uso de drogas que alteram a coagulação sangüínea.

2 .Se o seu uso decorreu de recomendação médica.

3 .Se os pacientes conhecem os seus possíveis efeitos indesejáveis.

4. Se o uso destas é relatado ao cirurgião.

5. Se o cirurgião pesquisa a sua utilização e se o mesmo recomenda a sua suspensão no pré-operatório.

6. O período em que o paciente suspendeu o uso das drogas.

\section{MÉTODO}

Trata-se de um estudo quantitativo com variáveis qualitativas, transversal, sobre o uso de medicamentos e/ou fitoterápicos, considerados como drogas, que alteram a coagulação sangüínea em pacientes passíveis de serem submetidos a um procedimento operatório eletivo em Taubaté.

O estudo foi desenvolvido no Hospital Universitário de Taubaté (HUT), no Hospital Regional de Taubaté ( HRT), e em um Consultório Particular de Cirurgia Plástica (Consultório). Foi aprovado previamente pelas Comissões de Ética dos respectivos hospitais.

O período da pesquisa, estipulado prévia e aleatoriamente pelos autores, foi de seis semanas entre os meses de setembro e novembro de 2003.

A pesquisa foi realizada por meio da distribuição de questionários aos pacientes em estudo, os quais foram previamente informados sobre o objetivo da mesma, inclusive do seu caráter sigiloso e, quando estavam de acordo, assinaram um termo de consentimento.

Foram analisados a idade, a escolaridade e o sexo. Foi perguntado se o paciente fez uso ou não nos últimos dez dias ou no último ano dos seguintes medicamentos: ácido acetilsalisílico (AAS) e vitamina E, e fitoterápicos como: ginkgo biloba, alho em comprimido, ginseng e gengibre. Em caso afirmativo, o mesmo deveria informar se os utilizou por recomendação médica ou não e se o cirurgião foi informado ou investigou o seu uso, ou recomendou suspendê-los por pelo menos uma semana antes da operação. Finalizando o questionário, perguntava-se ao paciente se ele tinha conhecimento da existência de algum efeito indesejável no uso destas substâncias.

Ressalta-se que no Hospital Regional de Taubaté (HRT) e no Consultório os pacientes receberam o questionário antes de serem examinados pelo médico, não podendo pois responder se informaram ao médico consultado naquela oportunidade sobre o uso das medicações e se o mesmo solicitou a sua suspensão.

O instrumento utilizado para análise estatística foi o Epi Info 2000 e para a elaboração das figuras o Microsoft Excel 2000.

\section{RESULTADOS}

Foram preenchidos 416 questionários, sendo 220 do HUT, 138 do HRT e 58 do Consultório. Excluíram-se três questionários do HUT e um do HRT em virtude da ausência de dados. Do total de 412 pacientes $(100 \%), 169(41,01 \%)$ referiram ter feito uso de alguma das drogas e 243 pacientes $(58,99 \%)$ não fizeram uso. A mediana das idades dos pacientes foi de 48 anos, sendo a mínima de 14 anos e a máxima de 92 anos. Observou-se que $62,72 \%(250)$ eram pacientes do sexo feminino e $37,27 \%$ (154) do sexo masculino. Nota-se que 58,99\%(243) dos pacientes pesquisados não fizeram uso das drogas questionadas. Entre os que as usaram, 39\%(160) o fez no último ano e 13,83\%(57) nos últimos dez dias. (Figura 1).

Observou-se que $28,15 \%$ (116) dos indivíduos pesquisados usaram AAS, 10,67\%(44) vitamina E, 5,58\%(23) ginkgo biloba, 5,33\%(22) gengibre, 1,94\%(08) ginseng e $1,21 \%$ (05) alho em comprimido.

Dentre os pacientes pesquisados no HUT(100\%:217), $31,25 \%$ (68) relatam ter feito uso de AAS, 6,25\%(14) de vitamina E, 5,8\%(13) de gengibre, 4,46\%(10) de ginkgo biloba, $1,78 \%(04)$ de alho em comprimido e $0.98 \%(02)$ de ginseng.

No HRT(100\%:137), 25,45\%(35) usou AAS, $10,94 \%$ (15) vitamina E, 5,1\%(07) gengibre, 2,18\%(03) ginkgo biloba, $2,18 \%(03)$ ginseng e $0,72 \%(01)$ alho em comprimido.

No Consultório(100\%:58) 25,86\%(15) utilizaram vitamina E, 18,96\%(11) AAS, 17,24\%(10) ginkgo biloba, 5,17\%(03) ginseng, 3,44\%(02) de gengibre e nenhum alho em comprimido. 


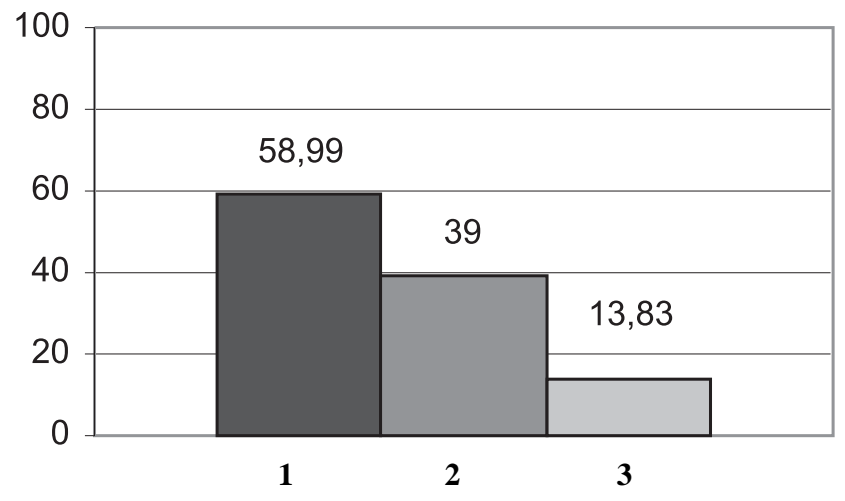

Figura 1 - Porcentagem de pacientes estudados em relação ao período de uso das drogas - Taubaté 2003.

Legenda: 1 - Não usaram; 2 - Último ano; 3 - Últimos 10 dias.

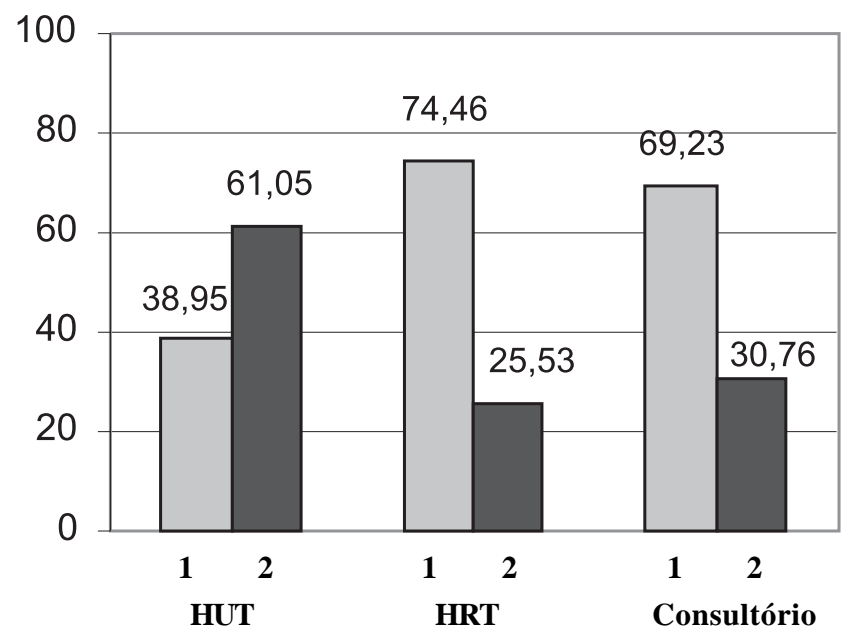

Figura 2 - Porcentagem dos pacientes que usaram as drogas por recomendação médica.

Legenda: 1 - Sim; 2 - Não.

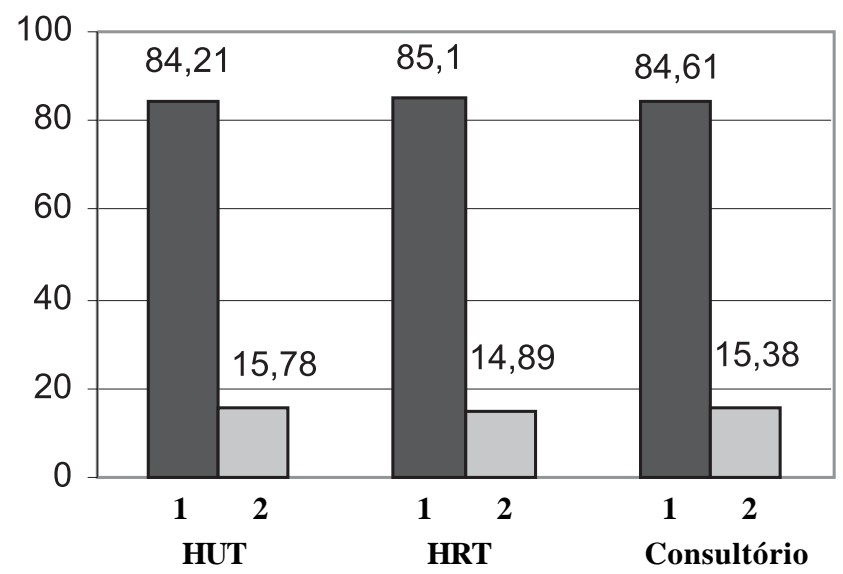

Figura 3 - Porcentagem de pacientes que usaram alguma das drogas em estudo e sabiam de eventuais efeitos colaterais. Legenda: 1 - Não; 2 - Sim.

Verificou-se no HUT, que 73,69\%(160) dos pacientes não receberam nenhuma orientação médica para interromper o uso das drogas estudadas no pré-operatório e que dentre os usuários de algumas drogas pesquisadas $26,31 \%$ informaram ao cirurgião sobre tal uso e $73,69 \%$ não.

Dentre os pacientes que utilizaram alguma das drogas em questão $38,95 \%$ no HUT, 74,46\% no HRT e $69,23 \%$ no Consultório o fizeram por recomendação médica (Figura 2).

Dentre os pacientes que fizeram uso prévio das drogas estudadas, observou-se que destes $15,78 \%$ no HUT, $14,89 \%$ no HRT e $15,38 \%$ no Consultório referiram possuir algum conhecimento dos possíveis efeitos colaterais provocados pelas mesmas (Figura 3).

\section{DISCUSSÃO}

O processo de coagulação foi descrito inicialmente por Morawitz, em 1905, como sendo constituído por duas fases principais: a formação de trombina e esta transformando fibrinogênio em fibrina. Segundo Langer et al, em 1964 MacFarlan, Davie e Ratnoff admitiram uma terceira fase, configurando o esquema de coagulação "em cascata" 28 .

A hemostasia necessita da atividade combinada de fatores vasculares, plaquetários e plasmáticos contrabalançados por mecanismos oponentes que limitam o acúmulo de plaquetas e fibrina na área de lesão vascular. São estes efeitos antagônicos que permitem ao sangue fluir dentro do vaso, sem coagular (trombose), nem extravasar (hemorragia).

Um grande número de fitoterápicos e medicamentos têm como efeito colateral interferir na coagulação sanguínea. Dentre as drogas questionadas neste estudo, 41,01\% (169) dos entrevistados afirmaram ter feito uso de alguma delas no período em questão, dado este semelhante ao estudo feito na Universidade do Colorado, EUA, no qual 50\% dos 500 pacientes pesquisados relataram o uso de fitoterápicos ou medicamentos alternativos dias antes da operação ${ }^{29}$.

No presente estudo observou-se uma porcentagem maior de mulheres, $(62,72 \%)$, que usaram alguma das drogas em questão, fato este verificado também por Laurence et al $(1999)^{30}$.

Dentre o total de pacientes estudados, $39 \%$ relataram uso no último ano, interrompendo-o em prazo superior a 10 dias antes da data da operação e $13,83 \%$ continuaram utilizando as drogas nos últimos 10 dias, sujeitando-se a maior risco de sangramento per e pós-operatório ${ }^{17}$ (Figura 1).

Neste estudo pode-se observar que o AAS foi o medicamento mais utilizado $(28,15 \%)$, talvez por ser o mais conhecido e prescrito com freqüência pelos médicos em virtude de seu papel no tratamento de doenças de alta prevalência, como as cardiovasculares e para analgesia e, provavelmente, pelo seu baixo custo ${ }^{26} \mathrm{e}$ estar disponível na rede pública. A vitamina $E$ foi o segundo medicamento mais usado $(10,67 \%)$, possivelmente por sua ação antioxidante ${ }^{24}$, demonstrada em um estudo onde reduz em $77 \%$ a morbidade e mortalidade de doenças arteriais coronarianas. A vitamina $\mathrm{E}$ teve maior utilização pelos pacientes do HRT em relação aos do HUT em 4\%, fato que poderia ser explicado pelo número de pacientes conveniados nesse serviço, portanto com possibilidade de adquirirem um medicamento de custo mais elevado.

No Consultório a vitamina E representou a droga mais utilizada seguido pelo AAS e ginkgo biloba. O maior poder 
aquisitivo destes pacientes também pode justificar a sua utilização.

O ginkgo biloba $(5,58 \%)$ e o gengibre $(5,33 \%)$ foram utilizados com porcentagens semelhantes O Ginseng e o Alho em comprimido foi utilizado por pequena porcentagem de pacientes.

Notou-se no HUT a ocorrência de uma elevada porcentagem $(73,69 \%)$ de pacientes que não foram informados pelos seus cirurgiões para suspender a medicação previamente ao procedimento cirúrgico. As eventuais complicações hemorrágicas intra e pós-operatórias ${ }^{17}$ provavelmente ocorreram em número reduzido,_pois somente $11,57 \%$ mantiveram a medicação "de moto próprio" nos últimos dez dias. Destacase assim que os cirurgiões que não orientaram pacientes a suspenderem as drogas no período pré-operatório, provavelmente não tinham conhecimento de seus possíveis efeitos adversos e, portanto não as correlacionaram com os eventuais sangramentos que possam ter ocorrido.

Verificou-se no HUT que a maioria dos pacientes $(73,69 \%)$ não informou ao cirurgião sobre o uso dos medicamentos e/ou fitoterápicos pesquisados, percentual este semelhante ao observado no estudo de Kaye et al (1999) ${ }^{31}$. Provavelmente essa omissão por parte dos pacientes ocorre porque os fitoterápicos não são considerados medicamentos pela população ${ }^{32}$ (Figura 3 ) .

Pode-se observar que $74,46 \%$ e $69,23 \%$ dos pacientes, no HRT e consultório respectivamente, afirmaram que o uso de alguma das drogas em questão foi feito por recomendação médica, e no HUT isto ocorreu em apenas 38,95\% (Figura 2). Ainda assim é preocupante a porcentagem dos indivíduos que ingerem estas drogas por decisão própria acreditando na inocuidade das mesmas e por isto não relatando o seu uso aos médicos. Em estudo realizado por Lawrence (1999), sobre o uso de fitoterápicos, $79 \%$ dos pacientes os usaram sem prescrição médica e deste total $36 \%$ o fizeram por recomendação de parentes ou amigos, $12 \%$ por propaganda em televisão e revistas, e $31 \%$ por "conta própria".
Em nossa casuística observou-se que mais de $80 \%$ dos pacientes, não tinham conhecimento dos eventuais malefícios que os medicamentos e fitoterápicos usados poderiam causar. Além dos problemas relacionados à coagulação sanguínea os pacientes desconhecem outros efeitos colaterais ${ }^{16}$. Chan, Chui e Lau ${ }^{33}$ em 2003, afirmaram que o ginseng pode induzir teratogenicidade em embriões de ratos, além de hipertensão, nervosismo e insônia. $\mathrm{O}$ ginkgo biloba pode causar alterações gastrointestinais leves e cefaléia. A hemorragia é um efeito colateral comum ao AAS, vitamina E, ginkgo biloba, alho, gengibre e ginseng, e, para o cirurgião, este é o efeito colateral mais importante e indesejável $^{34-40}$

\section{CONCLUSÕES}

Considerando o nosso estudo observamos que:

1. É elevada a porcentagem do uso das drogas que interferem na coagulação sanguínea, sendo as mais utilizadas o AAS, a vitamina E e o Ginkgo biloba. 2. No HRT e Consultório, a maior parte dos pacientes fez uso das drogas por recomendação médica 3 . A maioria dos pacientes pesquisados não tinha conhecimento dos efeitos colaterais destas drogas. 4. A maioria dos pacientes pesquisados não informou ao cirurgião a utilização destas drogas. 5. Na maior parte não houve preocupação dos médicos em se informar do uso e indicar a interrupção dos mesmos antes do procedimento cirúrgico. 6. Pequena porcentagem dos pacientes pesquisados continuou utilizando as drogas referentes nos dez dias que antecederam a cirurgia.

A análise dos dados acima nos permite concluir que é necessário investigar previamente o uso dessas drogas que potencialmente interferem na coagulação sanguínea e, se afirmativo, suspender até o $10^{\circ}$ dia de pré-operatório, com o objetivo de reduzir as eventuais complicações hemorrágicas no per e pós-operatórias, prevenindo possíveis implicações médicas e legais.

\footnotetext{
ABSTRACT

Background: To verify the frequency usage of nonprescription drugs and/or herbal medicines that coud interfere with blood coagulation. Also it is evaluated when this information has not been told to the surgeon. If this information had been known by the surgeon what attitude has been taken by the attending surgeon. Method: Four hundred and sixteen patients from the Hospital Universitário de Taubaté (HUT) in Regional Hospital de Taubaté (HRT) and in private plastic surgery clinic were studied on preoperative basis. A thorough search with quantitative and qualitative variables of nonprescription drugs (aspirin and E vitamin) and herbal medicines (Ginkgo biloba, Garlic and Ginger )was undertaken. Data were obtained though a questionnaire related to the use of these medications from one year and 10 days before surgery. It was computed if the surgeon has been informed of its use and what attitude had been taken about withdrawing or nor this medication. Results: Most of the patients (58.89\%) have not used any of these drugs, 39\% have used them the last year and $13.83 \%$ have used them in the last 10 days. Aspirin was the medicine most used and Ginkgo biloba was the preferred among the herbal medicines. The consumption of these drugs has been informed in 73.69\% of the patients that had used them. Conclusions: In the studied population it is high the percentage of the use of drugs that interferes in the blood coagulation. The most frequent medications are:Aspirin, $E$ vitamin and Ginkgo biloba. Most of the researched patients did not inform the surgeon about its use.It should be emphasized the need of questioning the use of these drugs before surgery, with the objective of reducing possible hemorrhage complications per and postoperatively, becauseof its medical complications and possible legal implications.
}

Key words: Phytotherapy; Plants, medicinal; Blood coagulation; Platelet aggregation inhibitors; Ginkgo biloba. 


\section{REFERÊNCIAS}

1. Bauer BA. Herbal therapy: what a clinician needs to know to counsel patients effectively. Mayo Clin Proc. 2000; 75(8):835-41.

2. Bateman J, Chapman RD, Simpson D. Possible toxicity of herbal remedies. Scott Med J. 1998; 43(1):7-15.

3. De Smet PA. Health risks of herbal remedies. Drug Saf. 1995; 13(2):81-93.

4. Brevoort P. The booming US botanical market: a new overview. Herbal Gram.1998; 44:33-46.

5. Ortenzi AV. Fitoterápicos e anestesia. Rev Soc Bras Anestesiol. 2001; 6:24-6.

6. Richard NS, Kowadlo N, Gibbs ME. Effect of the Ginkgo biloba extract, EGb 761, on memory formation in day-old chicks. Phamacol Biochem Behav. 2001; 69(3-4):351-8

7. Dergal JM, Gold JL, Laxer DA, Lee MS, Binns MA, Lanctot KL, Freedman M, Rochon PA. Potential interactions between herbal medicines and conventional drug therapies used by older adults attending a memory clinic. Drugs Aging. 2002; 19(11):879-86.

8. Rhee DJ, Katz LJ, Spaeth GL, Myers JS. Complementary and alternative medicine for glaucoma. Surv Ophthalmol. 2002; 46(1):43-55.

9. Lingaerde O, Foreland AR, Magnusson A. Can winter depression be prevented by Gingko biloba extract? A placebo-controlled trial. Acta Psychiatry Scand. 1999; 100(1):62-6.

10. Wong AH, Smith M, Boon HS. Herbal remedies in psychiatric practice. Arch Gen Psychiatry. 1998; 55(11):1033-44.

11. Akiba S, Kawauchi T, Oka T, Hashizume T, Sato T. Inhibitory effect of the leaf extract of Gingko biloba L. on oxidative stressinduced platelet aggregation. Biochem Mol Biol Int. 1998; 46(6): 1243-8.

12. Koch HP. Garlic. In: Lawson LD. The science and therapeutic application of allium sativum 1 and related species. 2th ed. Baltimore: William \& Wilkins; 1996.

13. Mar C, Bent S. An evidence-based review of the 10 most commonly used herbs. West J Med. 1999; 171(3):168-71.

14. Jie YH, Cammisuli S, Baggiolini M. Immunomodulatory effects of Panax Ginseng. C.A. Meyer in the mouse. Agents Actions. 1984; 15(3-4):386-91.

15. Singh VK, Agarwall SS, Gupta BM. Immunomodulatory activity of Panax ginseng extract. Planta Med. 1984; 50(6):462-5.

16. Hodges PJ, Kam PCA. The peri-operative implications of herbal medicines. Anesthesia. 2002; 57(9):889-99.

17. Ang-Lee MK, Moss J, Yuan CS. Herbal medicines and perioperative care. JAMA. 2001; 286(2):208-16.

18. Barrett B, Kiefer D, Rabago D. Assessing the risks and benefits of herbal medicine: an overview of scientific evidence. Altern Ther Health Med. 1999; 5(4):40-9.

19. Langner E, Greifenberg S, Gruenwald J. Ginger history and use. Adv Ther. 1998; 15(1):25-44.

20. Grant KL, Lutz RB. Ginger. Am J Health-Syst Pharm. 2000; 57(10):945-7.

21. Koo KL, Ammit AJ, Tran VH, Duke CC, Roufogalis BD. Gingerols and related analogues inhibit arachidonic acid-induced human platelet serotonin release and aggregation. Thromb Res. 2001; 103(5):387-97.

22. Carper J. Dez alimentos que combatem coágulos. In: Carper. Alimentos: o melhor remédio para a boa saúde. $5^{\text {a }}$ ed. Rio de Janeiro: Campos; 1995. p. 89.

23. Russell RM. Deficiência e excesso de vitaminas e oligominerais. In: Braunwald E, Fauci AS, Kasper DL, Hauser SL, Longo DL, Jameson JL, editors. Harrison medicina interna. $15^{\text {a }}$ ed. Rio de Janeiro: McGraw-Hil Interamericana do Brasil; 2002. p. 493-4.

24. Vassalo DV, Cunha V, Ressoni L. Fármacos em cardiologia. In: Cardiologia para o clínico geral. São Paulo: Atheneu; 1999. p. 591.
25. Qureshi AA, Qureshi N, Hasler-Rapacz JO, Weber FE, Chaudhary V, Crenshaw TD, et al. Dietary tocotrienols reduce concentrations of plasma cholesterol, apolipoprotein B, thromboxane B2, and platelet factor 4 in pigs with inherited hyperlipidemias. Am J Clin Nutr. 1991; 53(4 Suppl.):1042S-6S.

26. Lawrence C, Sakuntabhai A, Tiling-Grosse S. Effect of aspirin and nonsteroidal antiinflammatory drug therapy on bleeding complications in dermatologic surgical patients. JAmAcad Dermatol. 1994;31(6):988-92.

27. Lucker PW, Birkel M, Hey B, Loose I, Schaefer A. Pharmacokinetic interaction study of a fixed combination of $500 \mathrm{mg}$ acetylsalicylic acid/30mg pseudoephedrine versus each of the single active ingredients in healthy male volunteers Arzneimittelforschung. 2003; 53(4):260-5.

28. Langer B, Chamone DAF, Gomes MO. Bases dinâmicas da coagulação sanguínea. In: Langer B, Chamone DAF, Gomes MO. Coagulação e cirurgia. São Paulo: Sarvier; 1974. p.1.

29. Robb-Nicholson C. By the way, doctor. My doctor recently prescribed Zocor to treat my high cholesterol. She plans to test my liver for possible side effects, which makes me nervous. Is this a dangerous drug? What are its side effects? Harv Womens Health Watch. 2000; 7(10):8.

30. Tsen LC, Segal S, Pothier M, Bader AM. Alternative medicine use in presurgical patients. Anesthesiology, 2000, 93:148-51. Erratum in: Anesthesiology 2000; 93(5):1371.

31. Kaye AD, Clarke RC, Sabar R, Vig S, Dhawan KP, Hofbauer R, Kaye AM. Herbal medicines: current trends in anesthesiology practice- a hospital survey. J Clin Anesth. 2000; 12(6):468-71.

32. Blendon RJ, DesRoches CM, Benson JM, Brodie M, Altman DE. American's views on the use and regulation of dietary supplements. Arch Intern Med. 2001; 161(6):805-10.

33. Chan LY, Chiu PY, Lau TK. An in-vitro study of ginsenoside $\mathrm{Rb}_{1}$-induced teratogenicity using a whole rat embryo culture model. Hum Reprod. 2003; 18(10):2166-8.

34. Benjamin J, Muir T, Briggs K, Pentland B. A case of cerebral haemorrhage-can Ginkgo biloba be implicated? Postgrad Med J. 2001; 77(904):112-3.

35. Chang LK, Whitaker DC. The impact of herbal medicines on dermatologic surgery. Dermatol Surg. 2001; 27(8):759-63.

36. Destro MW, Speranzini MB, Cavalheiro Filho C, Destro T, Destro C. Bilateral haematoma after rhytidoplasty and blepharoplasty following chronic use of Ginkgo biloba. Br J Plast Surg. 2005; 58(1):100-1.

37. Fessenden JM, Wittenborn W, Clarke L. Ginkgo biloba: a case report of herbal medicine and bleeding postoperatively from a laparoscopic cholescystectomy. Ann Surg. 2002; 67(1):33-5.

38. Rose KD, Croissant PD, Parliamente CF, Levin MB. Spontaneous spinal epidural hematoma with associated platelet dysfunction from excessive garlic ingestion: a case report. Neurosurgery. 1990; 26(5):880-2.

39. Rowin J, Lewis SL. Spontaneous bilateral subdural hematomas associated with chronic Ginkgo biloba ingestion. Neurology. 1996; 46(6): 1775-6.

40. Smith IK. A dangerous mix. Anesthesiologists warn patients to stop taking herbal supplements before surgery. Time. 2000; 156(15):22.

Como citar este artigo:

Destro MWB, Speranzini MB, Destro C, Guerra C, Recco GC, Romagnolo LGC. Estudo da utilização no pré-operatório de medicamentos ou drogas fitoterápicas que alteram a coagulação sangüínea. Rev Col Bras Cir.[periódico na Internet] 2006 Mar-Abr;33(2). Disponível em URL: http://www.scielo.br/rcbc

Endereço para correspondência:

Dr.Marco Willians B. Destro

Rua Joaquim Tavares n ${ }^{\circ} 50$ - Centro - 12020-280 - Taubaté - SP

Tel:(012)3632-4244 e 3632-2407 Fax (012):3633-4903

E-mail: marcodestro@uol.com.br 